\section{Mesh-based Sensor Relocation for Coverage Maintenance in Mobile Sensor Networks}

Xu Li, Nicola Santoro SCS, Carleton University, Canada

and Ivan Stojmenovic SITE, University of Ottawa, Canada

\begin{tabular}{|c|}
\hline Sensor Relocation \\
\hline - Objective \\
- To replace failed sensors with redundant \\
mobile ones through autonomous node \\
movement. \\
- Evaluation criteria \\
- \# of message, storage load, total moving \\
distance and \# of moves.
\end{tabular}

\section{Discovering replacement}

Flooding-based [example] $\mathrm{M}=O\left(n^{\prime} n\right)$

- WCP [Wang, Cao, and Porta; 2004]

- Finding a redundant sensor for node replacement

Node relocation

- Moving the discovered redundant sensor to the position of a failed one
Quorum-based [example] $\quad \mathrm{M}=O\left(n^{\prime} \sqrt{n}\right)$

- WCPZ [Wang, Cao, Porta, and Zhang; 2005]

- ZONER [Li and Santoro; 2006]

Quorum formation requires cross network communication and generates inconstant per node storage load

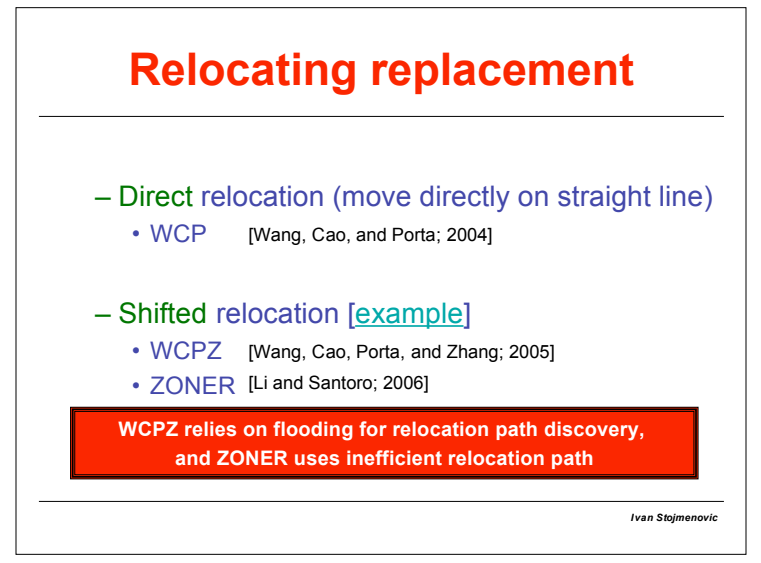

\section{A Mesh-based Sensor Relocation Protocol}

- A localized position based algorithm generating constant per node storage load.

- Requires no pre-knowledge of the sensor field.

- Uses near optimal relocation delay and balanced energy consumption.

- Guarantees nearby node replacement with very high probability, larger than $96 \%$. 

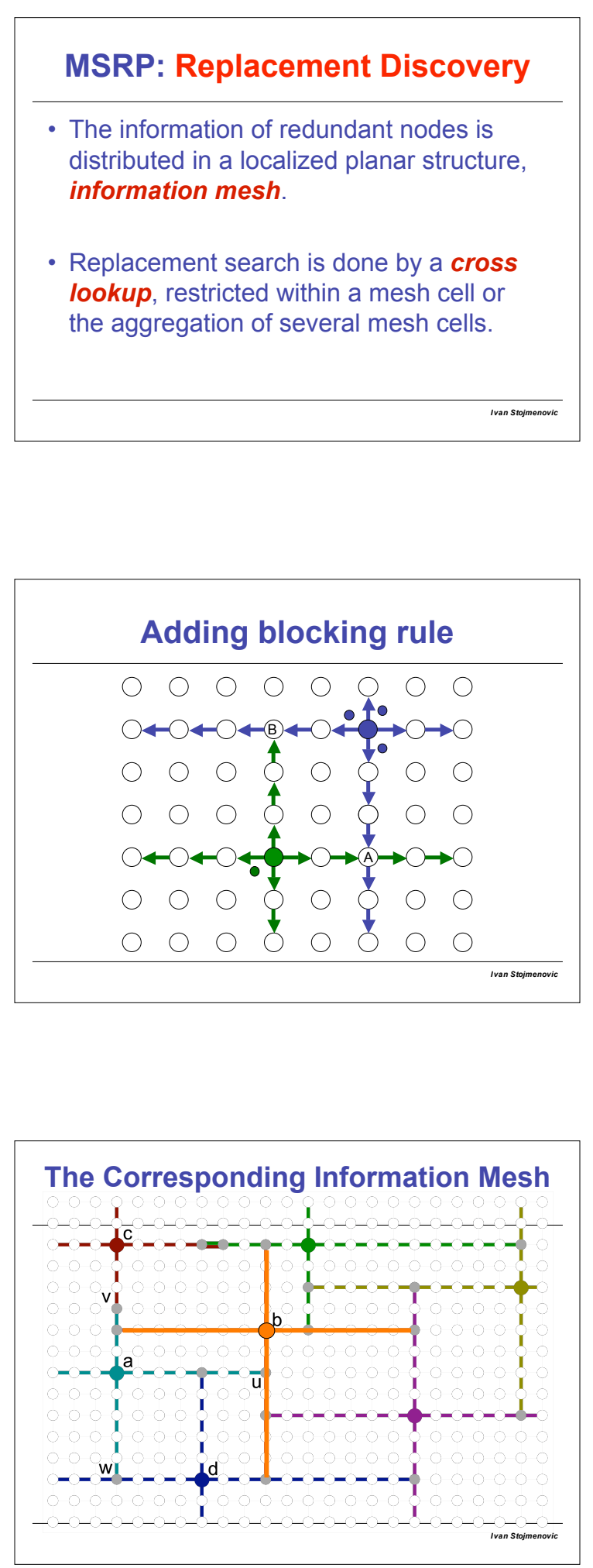
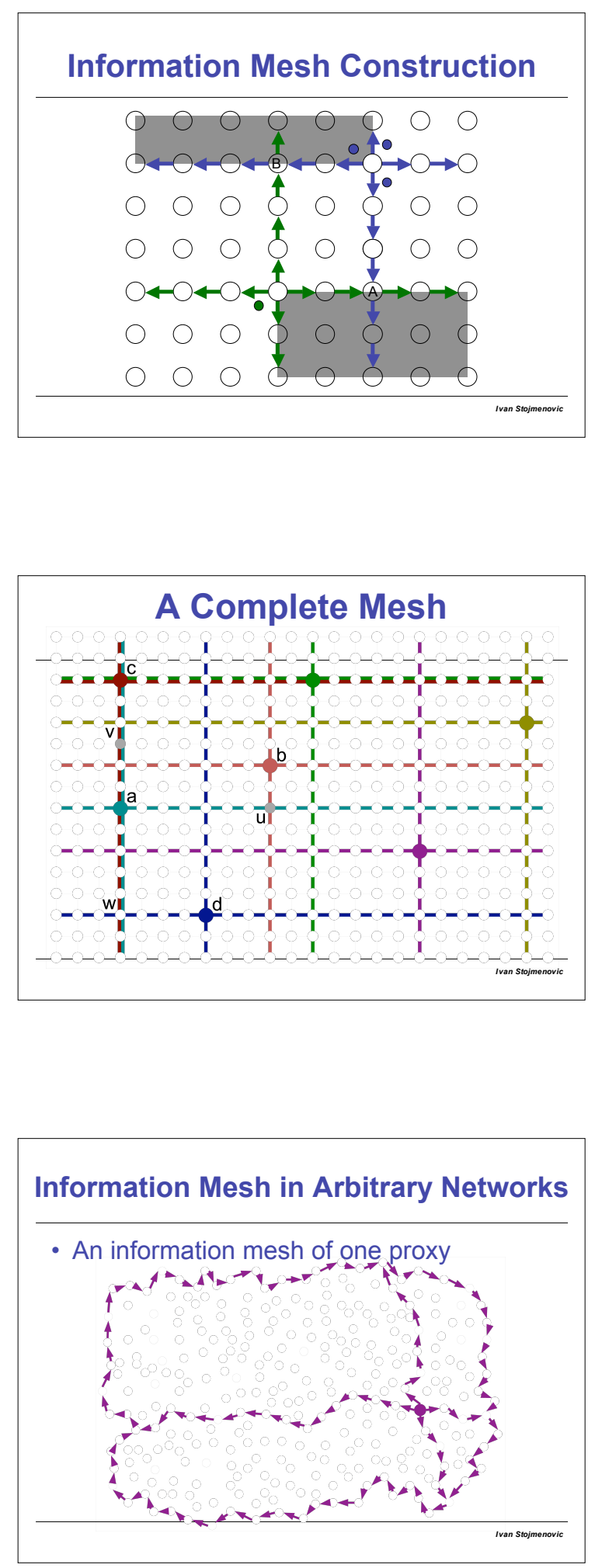

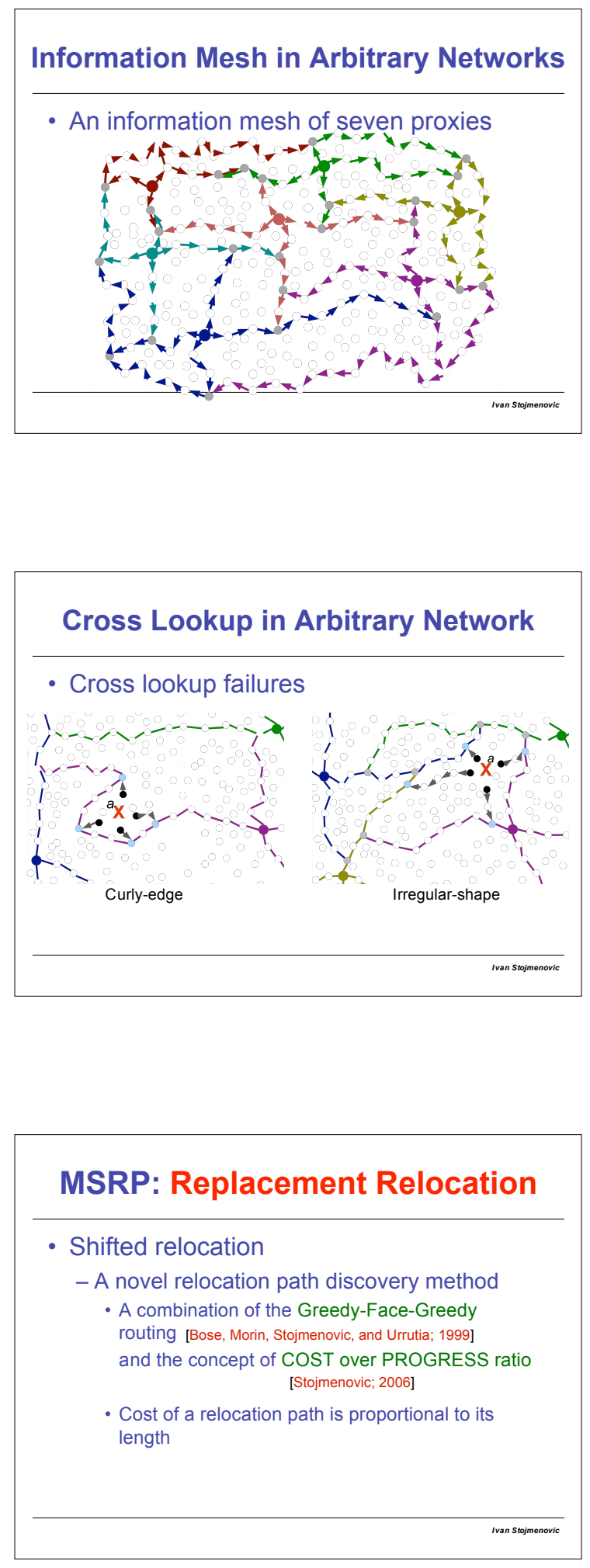
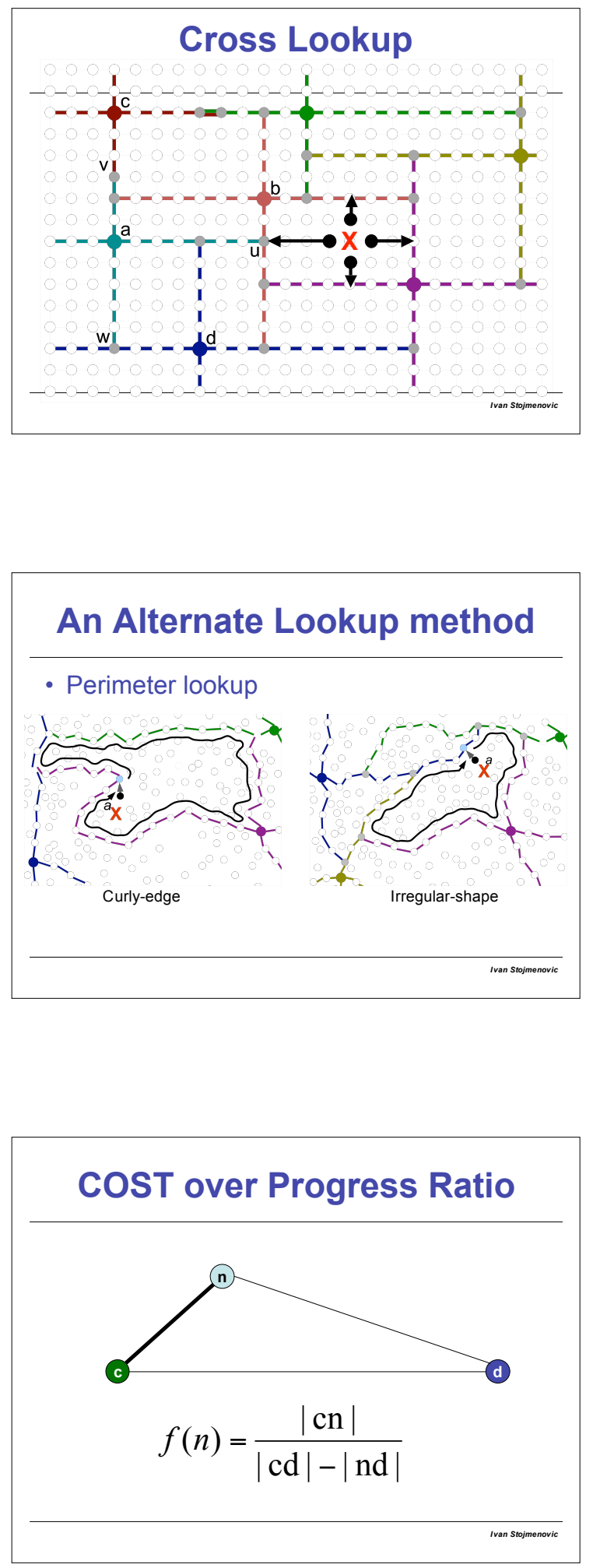


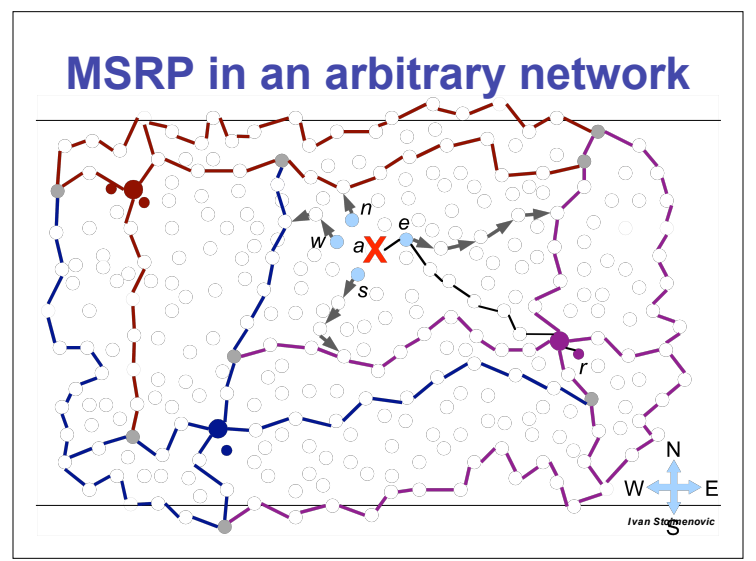

\section{Future Work}

- Finding closer replacement node in the worst and average case

- by allowing backward information propagation at blocking points (submitted)

- Voronoi diagram type of meshes ?

- Triangular or hexagonal meshes?

\section{References}

1. G. Wang, G. Cao, and T. L. Porta. "Proxy-Based Sensor Deployment for Mobile Sensor Networks". In Proc. of IEEE MASS, pp. 493-502, 2004.

2. G. Wang, G. Cao, T. L. Porta, and W. Zhang. "Sensor Relocation in Mobile Sensor Networks". In Proc. of IEEE INFOCOM, pp. 2302-2312, 2005.

3. X. Li and N. Santoro. "ZONER: A ZONE-based Senso Relocation Protocol for Mobile Sensor Networks". In Proc. of IEEE LCN/WLN, pp. 923-930, 2006.

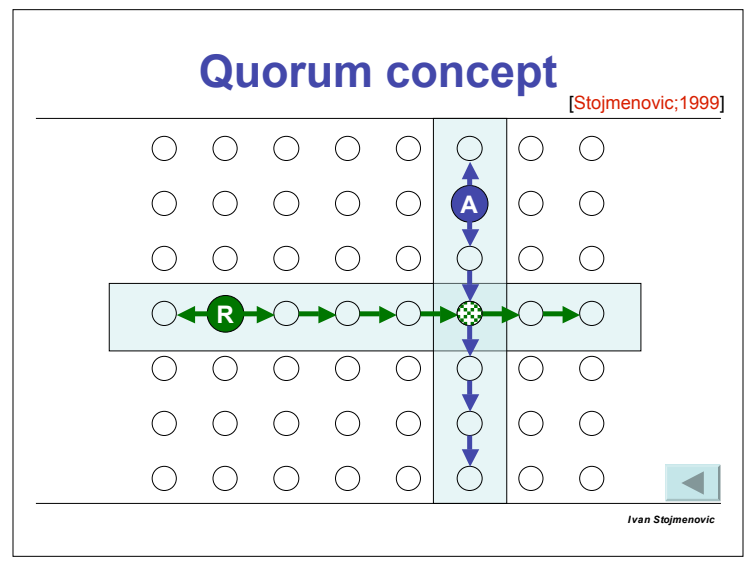

\section{Shifted node relocation}

- Find a path from replacement to the failed node and shift the position of the nodes along the path toward the failed node.

R

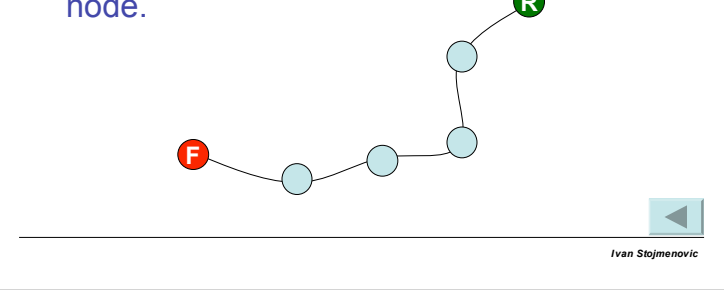




\section{Location service for sensor and actuator networks}

\author{
Ivan Stojmenovic
}

\section{Problem statements}

- Single actor/actuator/mobile sink moves in sensor networks

- Sensors are static

- Tradeoff between frequent reporting position and overhead for routing toward latest known position of actor (this problem elaborated here)

- Variant: several actors, each may report to neighboring sensors only, coordination among actors (ongoing research, see also relocation for some ideas)

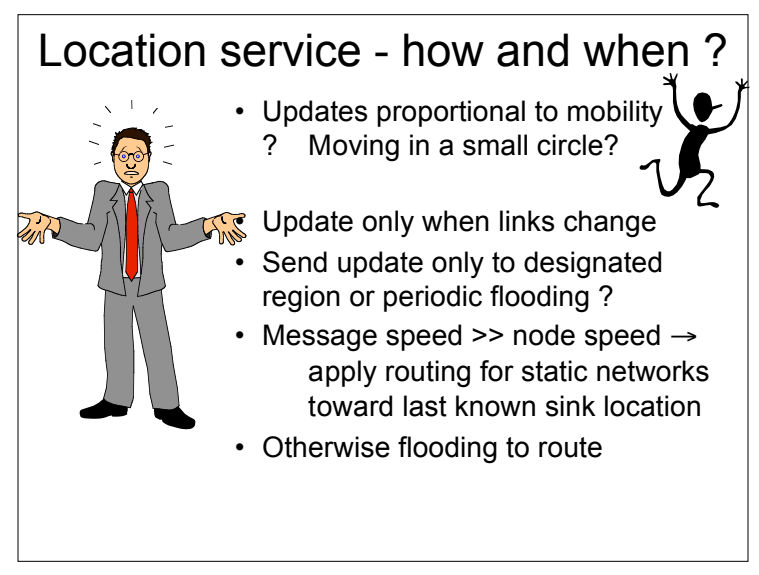

\section{Doubling circles routing}

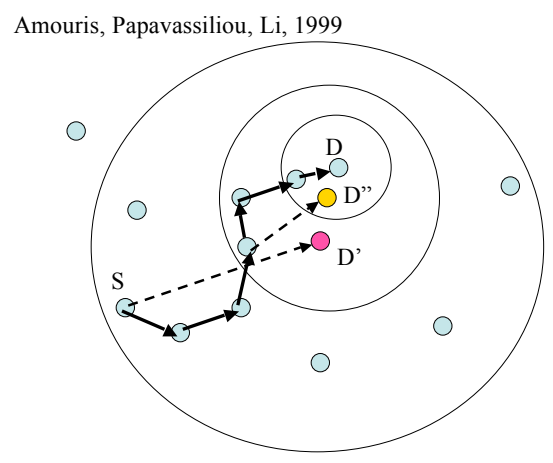

\section{Dynamic update}

- Dead-reckoning for mobile phones, Wolfson, Sistla '99:

- Report position, speed and direction of movement

- Use last known position, updated by reported movement, for sink position estimates

- Stojmenovic, Russell, Vukojevic 2000 for ad hoc networks 

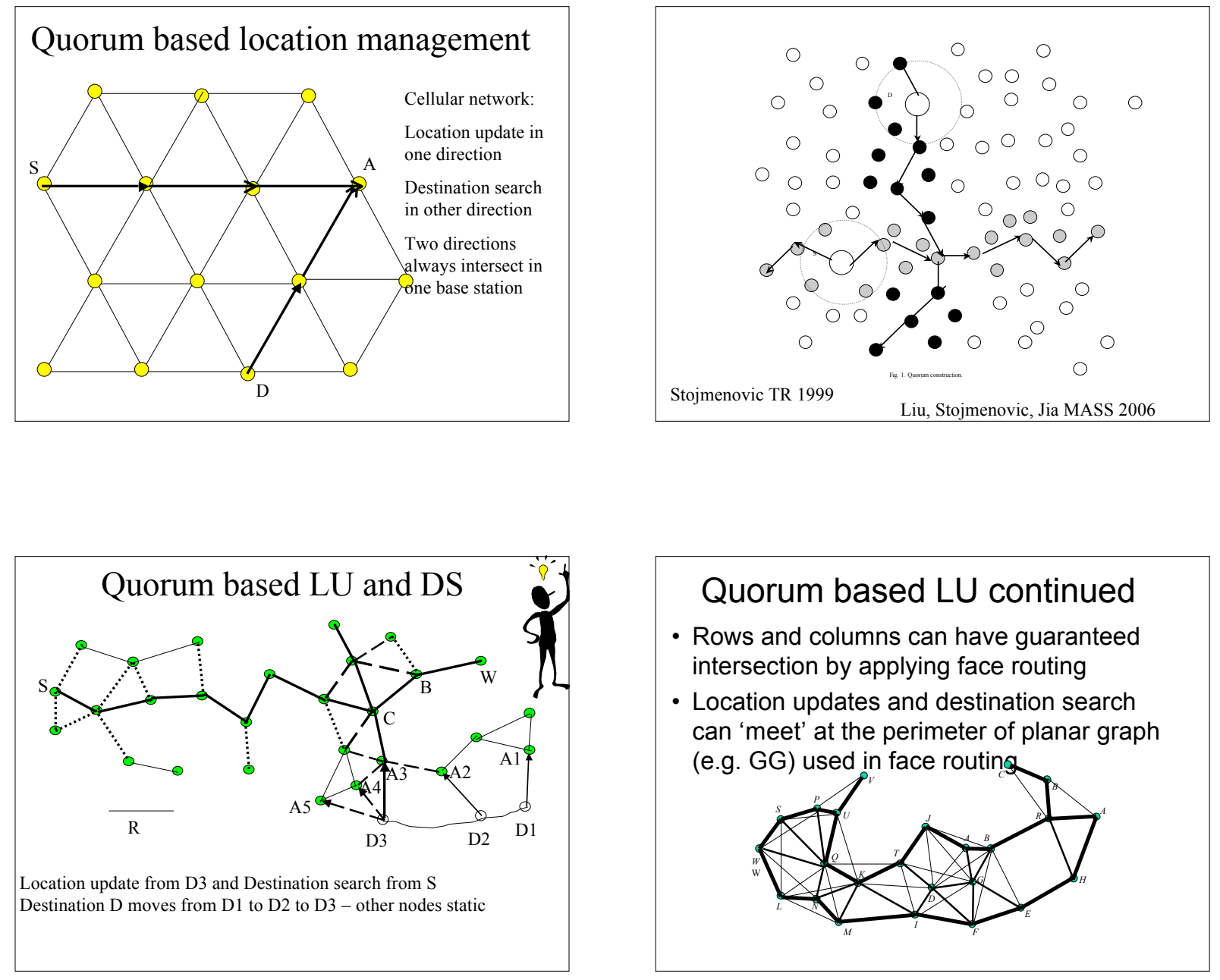

\section{Quorum based LU continued}

- Rows and columns can have guaranteed intersection by applying face routing

- Location updates and destination search can 'meet' at the perimeter of planar graph (e.g. GG) used in face routing

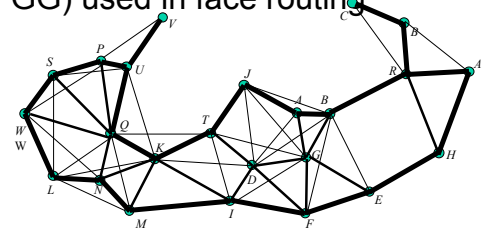

\section{Quorum - history}

- Ivan Stojmenovic, A routing strategy and quorum based location update scheme for ad hoc wireless networks, SITE, University of Ottawa, TR-99-09, September 1999

- Duplications (no citation):

- J. B. Tchakarov and N.H. Vaidya, Efficient content location in mobile ad hoc networks, IEEE Int. Conf. on Mobile Data Management MDM, 2004.

- I. Aydin and C.C. Shen, Facilitating match making service in ad hoc and sensor networks using pseudo quorum, 11th IEEE Int. Conf. Comp. Comm. Networks ICCCN, October 2002.

- Application and generalization (with citation):

- D. Niculescu and B. Nath, Trajectory based forwarding and its applications, Proc. ACM MOBICOM, San Diego, CA, Sept. 2003, 260-272.
} 

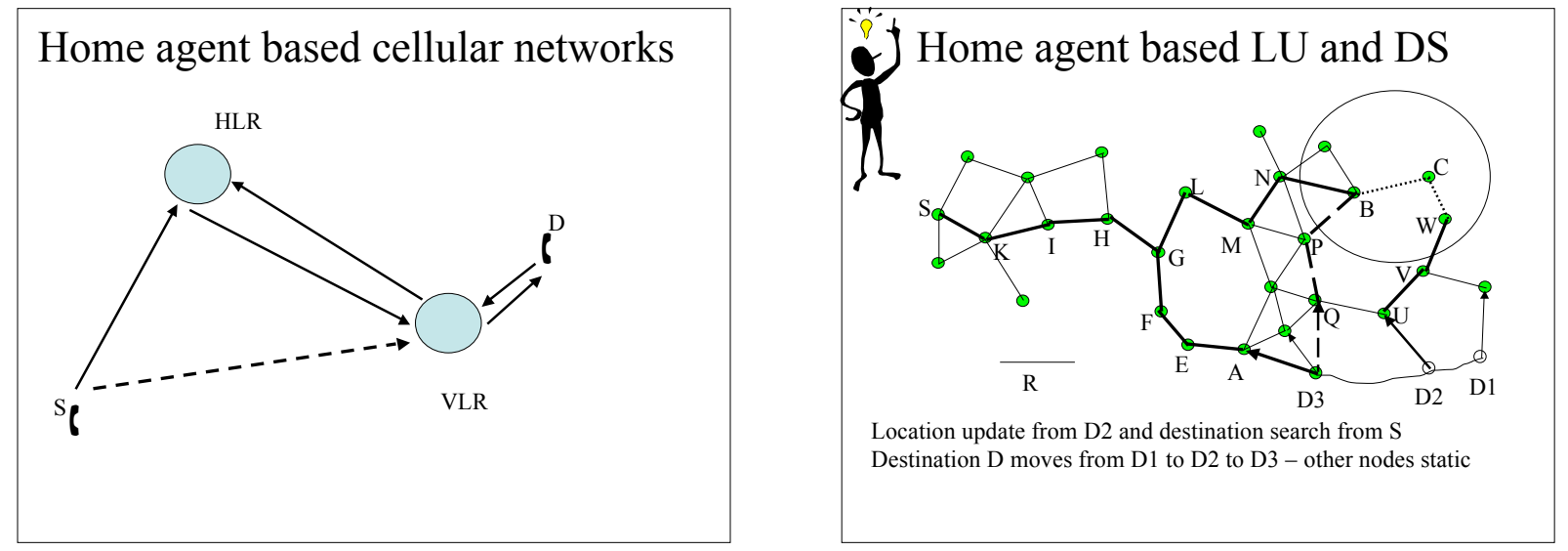

\section{Home agent based scheme - history}

- Stojmenovic, TR September 1999

- Woo and Singh, TR March 2000, Oregon State University; Wireless Networks, 7, 5, September 2001, 513-529.

- Blazevic, Buttyan, Capkun, Giordano, Hubaux and Le Boudec, TR, Swiss, Lausanne, December 2000;

IEEE Communication Magazine, June 2001.

- Morris, Jannotti, Kaashoek, Li, Decouto (MIT), $9^{\text {th }}$ ACM SIGOPS European Work., Kolding, Denmark, Sept. 2000.

- G. Pei and M. Gerla, Mobile Networks and Applications, 6, 4, August 2001, 331-337.

Data centric storage
- Ratnasamy, Estrin, Govindan, Karp, Shenker, Yin Yu 2002
- Geographic hash table
- Route data toward 'home' decided by hash
table and store there;
- 'home'= nearest sensor on the face containing
hashed location
- Find data by hashing and GFG routing toward
storage location

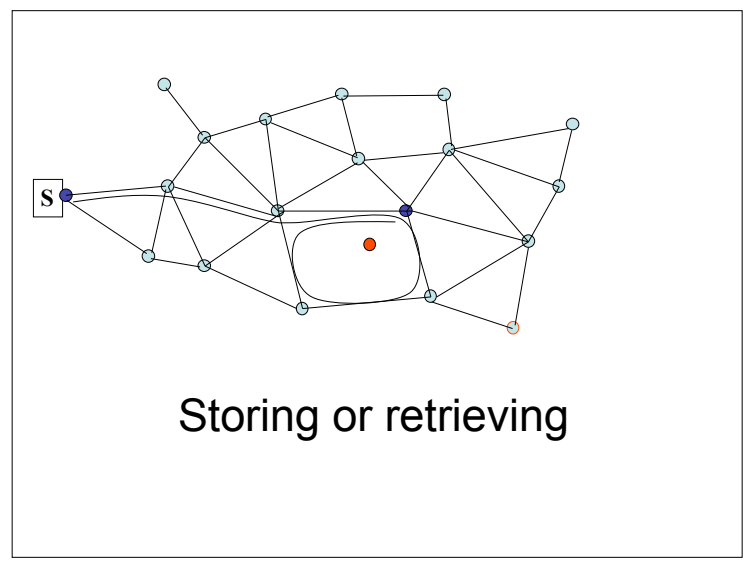

